\title{
Sensory conduction of the sural nerve in polyneuropathy ${ }^{1}$
}

\author{
DAVID BURKE, NEVELL F. SKUSE, AND A. KEITH LETHLEAN \\ From the Unit of Clinical Neurophysiology and the Division of Neurology, \\ The Prince Henry Hospital, Sydney, Australia
}

SYNOPSIS Using surface electrodes, sensory nerve action potentials (SAP) have been recorded in the proximal segment (mid-calf to lateral malleolus) and the distal segment (lateral malleolus to toe 5 ) of the sural nerve and in the median nerve in 79 control subjects. The values obtained for the distal segment of the sural nerve varied widely and in seven apparently normal subjects no SAP could be distinguished. In the proximal segment conduction velocities were over $40 \mathrm{~m} / \mathrm{s}$ and there was no significant change with age, unlike the median nerve in which a highly significant slowing occurred with age. Comparison of the results of sural and median sensory conduction studies in 300 consecutive patients screened for sensory polyneuropathy confirms the value of sural nerve sensory studies as a routine screening test, and confirms the belief that the changes in polyneuropathy are usually more prominent in lower limb nerves. It is therefore suggested that studies of sural sensory conduction form the single most useful test in the diagnosis of sensory polyneuropathy.

The value of sensory nerve conduction studies in the diagnosis of polyneuropathy is undisputed. However, in the diagnostic setting such studies are usually carried out only in upper limb nerves although the changes in polyneuropathy commonly present clinically first or more extensively in the lower limbs. Sensory conduction in lower limb nerves has been studied by a number of authors, the most recent and most detailed being that of Behse and Buchthal (1971), but, in general, these studies rely on needle electrodes, electronic averaging, and other technical refinements which put such studies beyond the technical capabilities of many diagnostic units and which therefore make it difficult to recommend them for use as a routine screening test.

Sensory conduction in the sural nerve may be studied without the need for complex techniques. Such studies have been reported in normal subjects and in patients with polyneuropathy by $\mathrm{Di}$ Benedetto $(1970,1972)$ and essentially similar but more extensive techniques have been used in this unit for the past five years. The sensory nerve action potential (SAP) may be readily and

1 Supported by a grant from the National Health and Medical Research Council of Australia. reliably obtained so that such studies provide data about the very nerve which may later be subjected to biopsy. This paper documents the results obtained in 79 control subjects in whom sensory conduction was studied in the proximal segment (mid-calf to lateral malleolus) and the distal segment (lateral malleolus to toe 5) of the sural nerve, and contrasts these results with those obtained in 300 consecutive patients in whom the tests were performed as screening procedures for sensory peripheral neuropathy. Sensory conduction of the sural nerve is contrasted with that of the median nerve to determine whether sural studies significantly increase the diagnostic yield in patients with symptoms which raise the possibility of sensory polyneuropathy.

\section{METHODS}

The control group consisted of 79 subjects aged between 8 years and 74 years. This group consisted of healthy normal volunteers (59 subjects), hospital patients suffering from a cerebral lesion (birth injury, tumour, or cerebrovascular disease) involving the ipsilateral cerebral hemisphere (seven subjects) and otherwise healthy patients with traumatic lesions or with symptoms of cervical spondylosis confined to a 
TABLE

VALUES FOR CONTROL SUBJECTS

\begin{tabular}{|c|c|c|c|c|c|c|c|}
\hline \multirow[t]{2}{*}{ Nerve and age range $(y r)$} & \multirow{2}{*}{$\begin{array}{l}\text { Subjects } \\
\text { (no.) }\end{array}$} & \multicolumn{3}{|c|}{$S A P(\mu V)$} & \multicolumn{3}{|c|}{ Conduction velocity $(\mathrm{m} / \mathrm{s})$} \\
\hline & & Range & Mean & $S D$ & Range & Mean & $S D$ \\
\hline $\begin{array}{l}\text { Sural (proximal segment) } \\
0-20 \\
21-40 \\
41-60 \\
61-80\end{array}$ & $\begin{array}{l}17 \\
26 \\
23 \\
13\end{array}$ & $\begin{array}{r}12-36 \\
9-32 \\
7-42 \\
6-18\end{array}$ & $\begin{array}{r}18 \cdot 4 \\
16 \cdot 4 \\
13 \cdot 6 \\
9 \cdot 8\end{array}$ & $\begin{array}{l}6 \cdot 4 \\
5 \cdot 5 \\
7 \cdot 5 \\
3 \cdot 6\end{array}$ & $\begin{array}{l}42 \cdot 4-52 \cdot 5 \\
40 \cdot 3-54 \cdot 7 \\
40 \cdot 6-53 \cdot 9 \\
40 \cdot 0-49 \cdot 3\end{array}$ & $\begin{array}{l}46 \cdot 9 \\
46 \cdot 2 \\
46 \cdot 4 \\
45 \cdot 5\end{array}$ & $\begin{array}{l}3 \cdot 0 \\
3 \cdot 7 \\
3 \cdot 7 \\
3 \cdot 1\end{array}$ \\
\hline $\begin{array}{l}\text { Sural (distal segment) } \\
0-20 \\
21-40 \\
41-60 \\
61-80\end{array}$ & $\begin{array}{c}16(17)^{*} \\
21(23) \\
19(21) \\
9(11)\end{array}$ & $\begin{array}{r}2-10 \cdot 5 \\
1 \cdot 5-7 \cdot 5 \\
1 \cdot 5-7 \cdot 5 \\
1 \cdot 5-5 \cdot 5\end{array}$ & $\begin{array}{l}3 \cdot 9 \\
3 \cdot 7 \\
3 \cdot 4 \\
3 \cdot 1\end{array}$ & $\begin{array}{l}2 \cdot 0 \\
1 \cdot 8 \\
1 \cdot 6 \\
1 \cdot 2\end{array}$ & $\begin{array}{l}31 \cdot 4-50 \cdot 0 \\
27 \cdot 1-42 \cdot 6 \\
28 \cdot 1-40 \cdot 4 \\
26 \cdot 2-40 \cdot 6\end{array}$ & $\begin{array}{l}36 \cdot 8 \\
33 \cdot 8 \\
34 \cdot 6 \\
32 \cdot 8\end{array}$ & $\begin{array}{l}4 \cdot 5 \\
3 \cdot 7 \\
3.9 \\
4 \cdot 9\end{array}$ \\
\hline $\begin{array}{r}\text { Median } \\
0-20 \\
21-40 \\
41-60 \\
61-80\end{array}$ & $\begin{array}{l}17 \\
25 \\
21 \\
12\end{array}$ & $\begin{array}{l}16-28 \cdot 5 \\
12-36 \\
10-60 \\
7 \cdot 5-26\end{array}$ & $\begin{array}{l}22 \cdot 4 \\
22 \cdot 5 \\
20 \cdot 1 \\
14 \cdot 0\end{array}$ & $\begin{array}{r}3 \cdot 6 \\
8 \cdot 0 \\
12 \cdot 8 \\
5 \cdot 2\end{array}$ & $\begin{array}{l}46 \cdot 2-61 \cdot 5 \\
44 \cdot 0-61 \cdot 0 \\
42 \cdot 3-58 \cdot 5 \\
43 \cdot 3-58 \cdot 4\end{array}$ & $\begin{array}{l}54 \cdot 9 \\
54 \cdot 5 \\
51 \cdot 2 \\
49 \cdot 4\end{array}$ & $\begin{array}{l}5 \cdot 7 \\
4 \cdot 9 \\
4 \cdot 7 \\
4 \cdot 1\end{array}$ \\
\hline
\end{tabular}

* For the distal segment of the sural nerve calculations are based only on those in whom an SAP was found-that is, 65 of 72 subjects. The numbers in parentheses are the total numbers tested.

limb contralateral to that studied (11 and two subjects respectively). Control subjects were questioned to elicit sensory symptoms, the presence of diabetes mellitus, alcoholism, and other such disorders, were examined for evidence of polyneuropathy, and were excluded from the control group where the possibility of peripheral nerve pathology existed. The patient group consisted of 300 consecutive patients in whom both median and sural nerve conduction values were determined as a screening test for polyneuropathy. Patients suffering clinically from bilateral carpal tunnel syndrome or mononeuritis multiplex were excluded.

Standard neurophysiological techniques for stimulation and recording were used (Preswick and Jeremy, 1964). Parameters measured were peak to peak SAP amplitude and latency to the onset of the negative phase. Conduction 'velocity' was obtained by dividing latency by conduction distance. The orthodromic :SAP of the median nerve was recorded at the wrist :after stimulation of the index finger with ring electrodes. To elicit the 'proximal sural SAP' bipolar surface electrodes were placed on the posterior aspect of the calf at the junction of the middle and lower thirds of the leg slightly lateral of midline. In this position the distance from the lateral malleolus varied from 12 to $18 \mathrm{~cm}$ in different patients. A second set of electrodes was placed behind and below the lateral malleolus over the sural nerve which is usually palpable at this site. Both antidromic and orthodromic recordings of the SAP were made. To elicit the 'distal sural SAP' the nerve was stimulated at the lateral malleolus and the antidromic SAP was

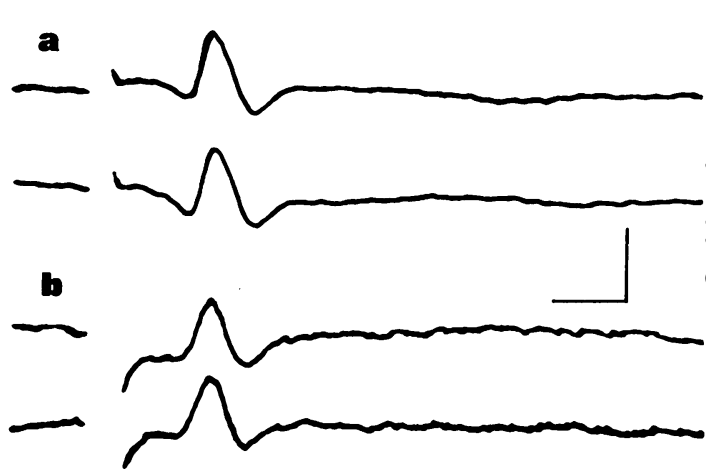

FIG. 1. SAP of the proximal segment of the sural nerve: (a) antidromic, (b) orthodromic. Calibrations: vertical (a) $30 \mu \mathrm{V}$, (b) $15 \mu \mathrm{V}$; horizontal $2 \mathrm{~ms}$.

recorded by bipolar electrodes applied to the lateral edge of the dorsum of the foot with the active electrode immediately proximal to the fifth metatarsophalangeal joint.

The studies were carried out in an air-conditioned room at constant temperature. No systematic recording of limb temperature was made, skin temperature being determined in only some subjects. As a routine, where a limb felt cool it was warmed by immersion in warm to hot water.

\section{RESULTS}

CONTROL SUBJECTS The results obtained are 


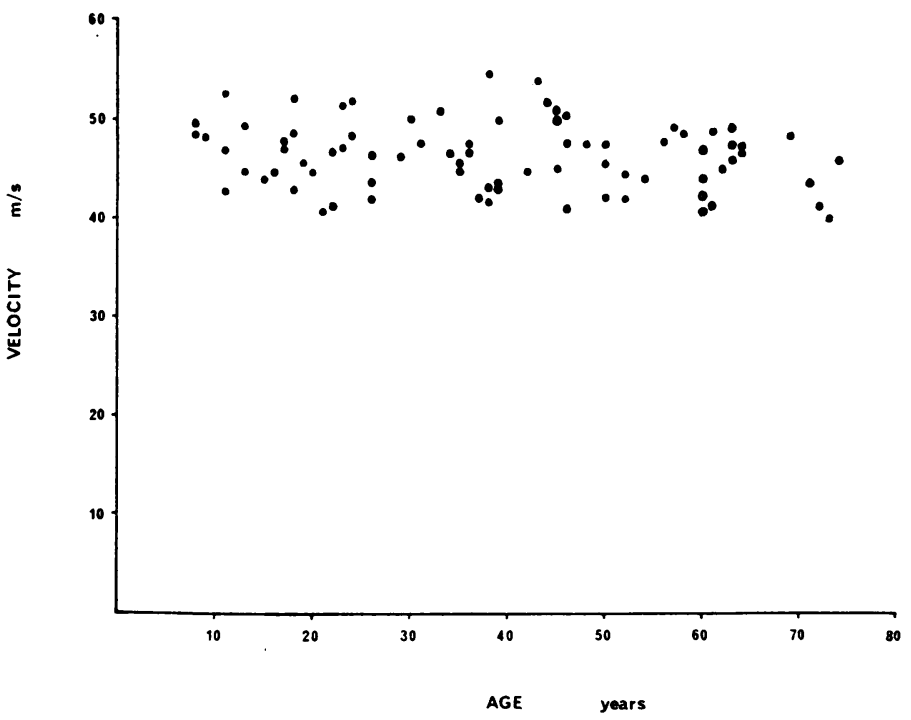

FIG. 2. Sensory conduction velocity of the proximal segment of the sural nerve. Velocity changes little with age.

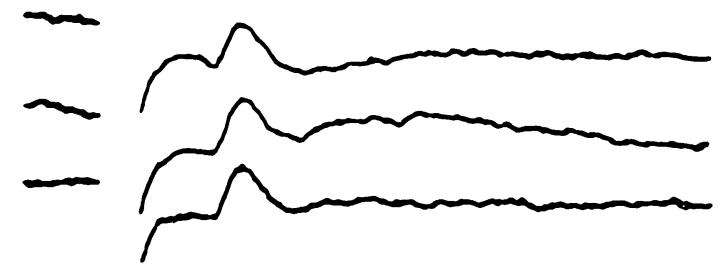

FIG. 3. SAP of the distal segment of the sural nerve. Calibrations: vertical $15 \mu \mathrm{V}$, horizontal $2 \mathrm{~ms}$.

detailed in the Table. In the proximal segment of the sural nerve the antidromic SAP was usually of higher amplitude than the orthodromic SAP (Fig. 1) and was therefore used for amplitude measurements. SAP amplitude decreased greatly with age, the differences between the age group 0-20 years and the age group 61-80 years being highly significant $(\mathrm{P}<0.0005)$. There was a slight tendency for decreasing velocity with increasing age (Fig. 2) but this was not prominent, and there was no significant difference between the values obtained in the $0-20$ year age group and the $61-80$ age group $(0 \cdot 15>P>0 \cdot 10)$. The lower limit of normal sensory conduction velocity for the proximal segment of the sural nerve may be considered to be $40.0 \mathrm{~m} / \mathrm{s}$ for each group.

The distal sural SAP was generally of small amplitude (Fig. 3) and in seven subjects (aged 16, $38,38,54,57,64$, and 72 years) the SAP could not be recognized. Within each age group the scatter of amplitude and velocity measurements was large, obscuring somewhat the changes with age.

In the median nerve both SAP amplitude and conduction velocity decreased with increasing age, the differences between the 0-20 year and the $61-80$ year age group being highly significant $(\mathrm{P}<0.0005$ and 0.005 respectively). The lower limits of normal median nerve sensory conduction velocity have been taken as $45 \mathrm{~m} / \mathrm{s}$ up to 40 years and $42 \mathrm{~m} / \mathrm{s}$ for ages over 40 years.

PATIENTS SCREENED FOR POLYNEUROPATHY The sensory conduction velocities are plotted in Figs 4-7 for the four age groups, with proximal sural values along the $\mathrm{Y}$-axis and corresponding median values on the $\mathrm{X}$-axis. The horizontal line in each Figure represents the lower limit of normal proximal sural sensory velocity $(40 \mathrm{~m} / \mathrm{s})$ and the vertical line represents the lower limit of normal median sensory velocity. With surface electrodes a SAP was not recorded with a conduction velocity below $25-30 \mathrm{~m} / \mathrm{s}$. Such SAP were presumably so dispersed that their amplitudes could not be distinguished from noise in single sweeps. In some patients use of needle electrodes and an input transformer has allowed identification of a low amplitude SAP which on occasions conducted at near normal rates. 


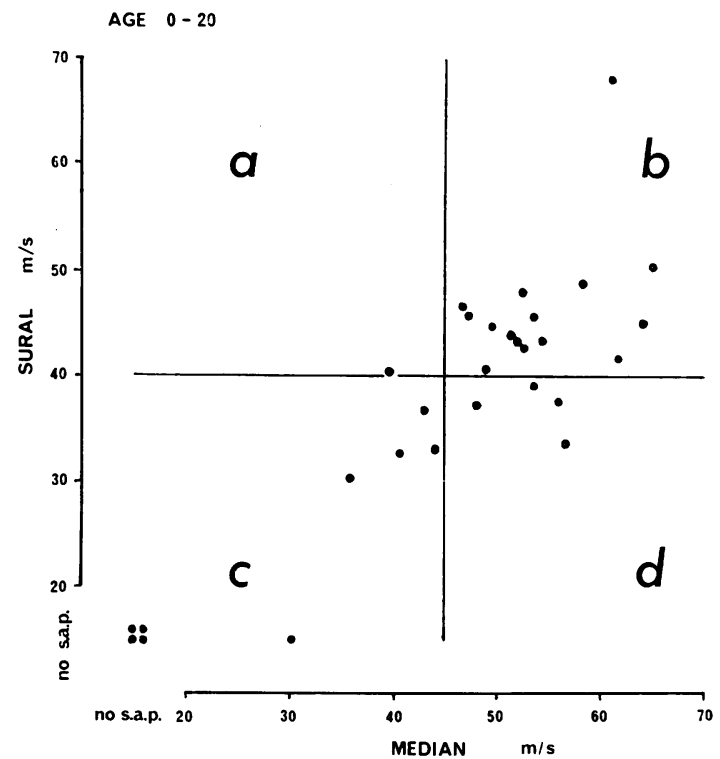

FIG. 4. Proximal sural and median sensory velocities in patients aged between 7 and 20 years. As in Figs 5, 6 , and 7 , the vertical line represents the lower limit of normal median sensory velocity and the horizontal line the lower limit of normal proximal sural velocity. These lines divide the graphs into four quadrants labelled $\mathrm{a}-\mathrm{d}$, but for the sake of clarity the quadrants have been so labelled only in this Figure.

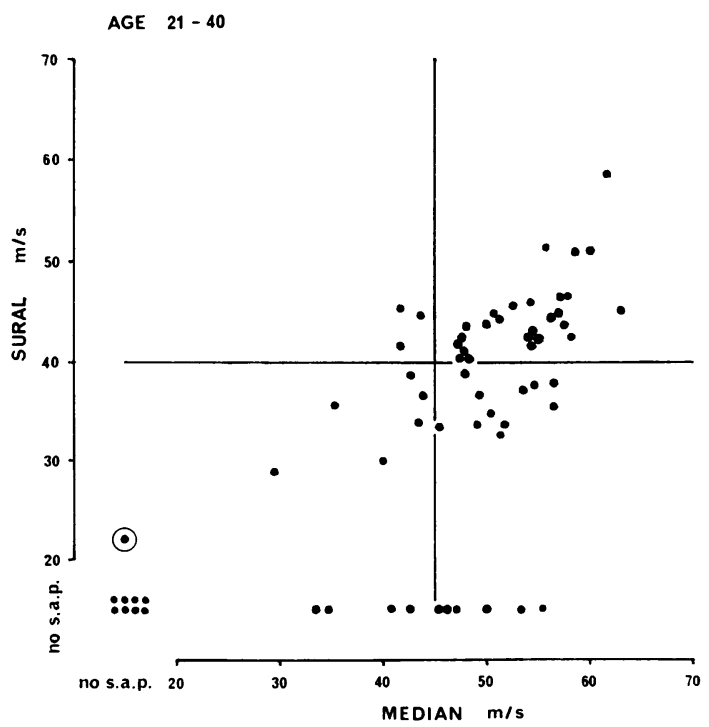

FIG. 5. Proximal sural and median sensory velocities in patients aged 21-40 years. Seventeen patients had abnormal sural studies but normal median studies. The reverse occurred in only three subjects.

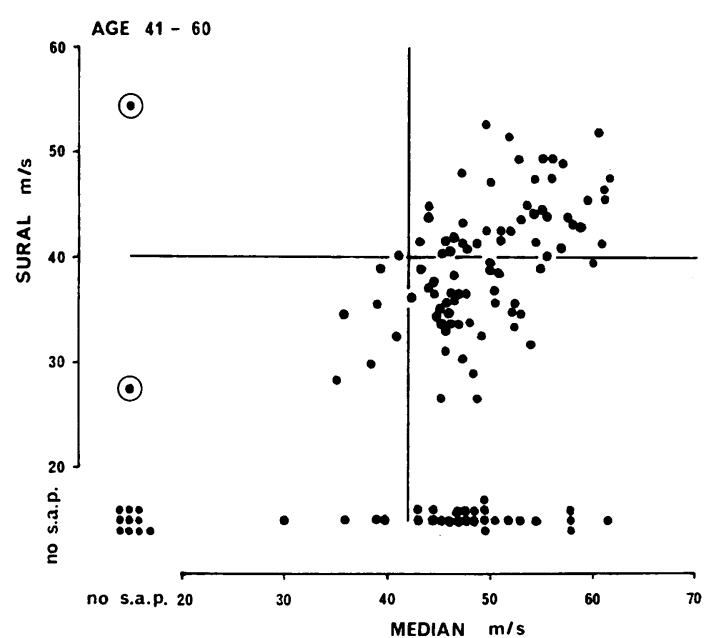

FIG. 6. Proximal sural and median sensory velocities in patients aged 41-60 years. Sixty-one patients had abnormal sural studies but normal median studies. The reverse occurred in only two patients.

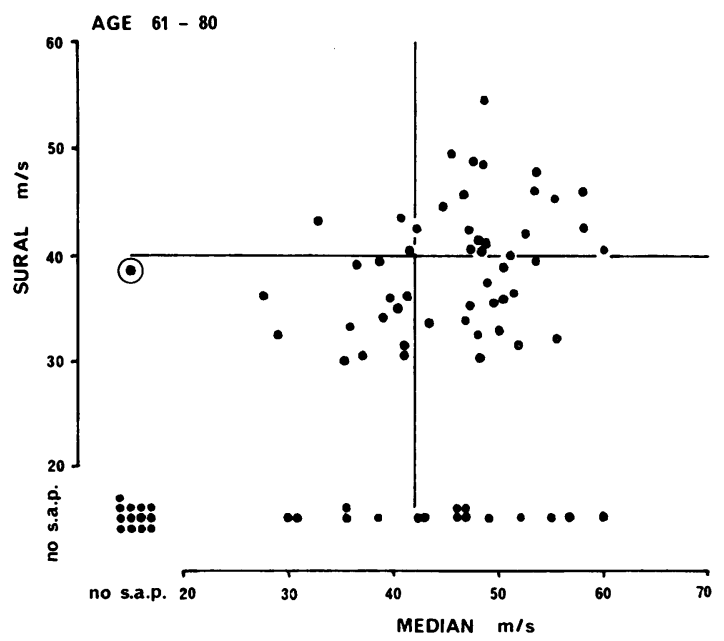

FIG. 7. Proximal sural and median sensory velocities in patients aged 61-80 years. Twenty-five patients had abnormal sural studies but normal median studies. The reverse occurred in only three patients. 
A comparison of quadrants ' $a$ ' and ' $d$ ' of Figs 4-7 reveals that electrical evidence of neuropathic change is more common and more extensive in the lower limb sensory nerve. Few patients fall into quadrant ' $a$ ' with normal sural and abnormal median studies (total of nine patients), but the reverse is common such that a total of 107 patients fall into quadrant ' $d$ '. The presence of greater abnormality in the median values than in sural values might be expected to occur in some patients because the carpal tunnel is a common site of nerve compression, but no such factor can be invoked to explain the greater frequency and severity of abnormality in the sural nerve.

In the four subjects whose values are circled in Figs 5, 6, and 7, a median SAP could not be recorded, although a sural SAP (of less than normal amplitude in three of the four) was recorded with conduction values ranging from 22 to $53 \mathrm{~m} / \mathrm{s}$. Clinically, these patients suffered from the Landry-Guillain-Barré-Strohl syndrome (two patients) and acute relapsing polyneuropathy (two cases). One of the two latter was subjected to sural nerve biopsy at a time when the sural sensory conduction values were still within normal limits. Histological findings were within normal limits.

Proximal sural conduction velocity was within normal limits in 112 patients. Of these, 21 had SAP amplitudes below acceptable levels. Many patients with conduction velocities just below the normal range had SAPs of abnormal amplitude and shape, and it is in these patients that amplitude measurements proved most useful, as confirmation of the presence of sensory abnormality.

Determinations of sensory conduction in the distal segment of the sural nerve have been performed in 98 patients. Using the technique described, a distal sural SAP has not been recorded when the proximal sural SAP could not be distinguished, was of lower than normal amplitude, or was conducted with abnormally slow velocity-that is, when there has been abnormality of sensory conduction in the proximal segment of the sural nerve, a SAP has not been recordable in the distal segment despite painstaking effort.

\section{DISCUSSION}

Satisfactory studies of sensory conduction in the proximal segment of the sural nerve can be carried out without difficulty in all normal patients with the same standard techniques as used for upper limb nerves. Of all lower limb sensory nerves only the sural provides such a ready index of lower limb sensory function. There can be little dispute that sensory conduction studies in lower limb nerves have a higher percentage yield in polyneuropathy than such studies in upper limb nerves. In view of the high number of normal median sensory velocities in patients with an abnormal sural SAP, and in view of the ease with which sural conduction may be studied, there appears to be little place for using median sensory studies as the sole criterion of sensory polyneuropathy.

The relatively slight changes with age in sural conduction velocity contrast sharply with the changes in sural SAP amplitude. A decrease in amplitude may be attributed not only to changes in skin texture with age but also to pathological changes within the nerve which are acceptable as within normal limits for age. It can be argued that with larger samples significant conduction slowing would have been demonstrable in the elderly group, but the samples were sufficiently large to demonstrate a highly significant slowing of median sensory velocity in the same patients. This suggests that either age changes are more prominent in the upper than the lower limbs, or that a factor other than age affects median nerve function but not sural nerve function. Such a factor could be minor trauma or subclinical compression of the median nerve as it passes through the carpal tunnel, and this provides a further rationale for recommending tests of sural sensory conduction in polyneuropathy.

The place of studies of distal sural sensory conduction is unclear, since the SAP cannot be readily obtained in all apparently normal controls. It is possible that the inability to record a distal sural SAP with surface electrodes may represent early changes of an as yet subclinical neuropathy, but in all probability it resulted from the difficulty in distinguishing a low amplitude SAP from noise. Thus, it cannot be maintained that absence of the distal sural SAP implies neuropathic abnormality. However, the 
presence of a distal sural SAP with the technique described here, while not excluding nerve pathology, probably excludes a clinically significant sensory polyneuropathy.

\section{REFERENCES}

Behse, F., and Buchthal, F. (1971). Normal sensory conduc- tion in the nerves of the leg in man. Journal of Neurology, Neurosurgery, and Psychiatry, 34, 404-414.

Di Benedetto, M. (1970). Sensory nerve conduction in lower extremities. Archives of Physical Medicine and Rehabilitation, 51, 253-258.

Di Benedetto, M. (1972). Evoked sensory potentials in peripheral neuropathy. Archives of Physical Medicine and Rehabilitation, 53, 126-131 and 134.

Preswick, G., and Jeremy, D. (1964). Subclinical polyneuropathy in renal insufficiency. Lancet, 2, 731-732. 